Recherches en didactique des langues et des cultures

Les cahiers de l'Acedle

12-1 | 2015

Prendre la diversité au sérieux en didactique / didactologie des langues

\title{
Quelles approches didactiques pour le FLE en Australie occidentale?
}

\section{Céline Doucet et Edith Cowan}

\section{OpenEdition}

Journals

Édition électronique

URL : http://journals.openedition.org/rdlc/345

DOI : $10.4000 /$ rdlc.345

ISSN : $1958-5772$

Éditeur

ACEDLE

Référence électronique

Céline Doucet et Edith Cowan, «Quelles approches didactiques pour le FLE en Australie occidentale? ", Recherches en didactique des langues et des cultures [En ligne], 12-1 | 2015, mis en ligne le 01 juin 2015, consulté le 25 juin 2020. URL : http://journals.openedition.org/rdlc/345 ; DOI : https://doi.org/ $10.4000 /$ rdlc.345

Ce document a été généré automatiquement le 25 juin 2020.

\section{(i) $\ominus$

Recherches en didactique des langues et des cultures is licensed under a Creative Commons AttributionNonCommercial-NoDerivatives 4.0 International License 


\title{
Quelles approches didactiques pour le FLE en Australie occidentale?
}

\author{
Céline Doucet et Edith Cowan
}

\section{Introduction}

1 Les profils des publics adultes apprenant le français se sont diversifiés au fil du temps dans un contexte de mondialisation. Ils sont variés (public étudiant, migrant, professionnel, spécialiste non francophone), ont des ressources linguistiques multiples et leurs objectifs d'apprentissage concernent aussi bien les domaines professionnels que personnels. L'offre de formation s'est adaptée en proposant des cours de français sur objectifs universitaires (FOU), français sur objectifs spécifiques (français du tourisme, etc.) français pour migrants...

2 En Australie Occidentale, il existe une demande forte en matière d'apprentissage du français à l'Alliance française de Perth ${ }^{1}$ de la part d'un public adulte ayant des caractéristiques variées. La question est de savoir, dans ce contexte particulier, comment l'offre répond à la diversification des demandes et des projets avec le français. Quels moyens sont mis en œuvre par l'Alliance française pour articuler la/les demande(s) et les démarches didactiques choisies en matière d'enseignement du français?

3 Notamment, bien que le développement d'une compétence communicative constitue un objectif massivement dominant du point de vue de l'offre, on peut se demander si cela coïncide avec la demande, autrement dit, si l'intérêt pour le français et pour son apprentissage se traduit forcément par l'envie d'acquérir une compétence communicative en langue française, ce qui soulève, entre autres, la problématique plus générale de la contextualisation de l'enseignement du FLE.

4 Cette recherche interprétative traite de la prise en compte ou non de la diversité des apprenants et de leurs besoins et va mettre en évidence, par un jeu de regards croisés, un certain conflit entre la demande et l'offre en matière d'apprentissage/enseignement du FLE dans ce contexte particulier. 
Dans un premier temps, nous proposerons quelques informations sociolinguistiques sur le français en Australie, sur les représentations qu'en ont les Australiens puis sur la place du français dans le monde éducatif ouest- australien. Nous analyserons ensuite les discours des apprenants que nous confronterons aux orientations didactiques mises en œuvre pour l'enseignement $d u$ français. Nous interrogerons, dans un troisième temps, les raisons des divergences constatées.

$6 \quad$ Notre article s'appuie sur des enquêtes de terrain menées dans le cadre d'un doctorat sur la contextualisation de l'enseignement du FLE dans des situations contrastées (Louisiane, Australie). Cette recherche qualitative rassemble des données issues de questionnaires et d'entretiens auprès d'apprenants, d'enseignants de français et d'acteurs sociaux concernés par l'enseignement du français dans des structures éducatives variées en Australie Occidentale.

7 Ne seront évoquées ici que les enquêtes réalisées dans une Alliance française, conduites sous forme de questionnaires de types variés et d'entretiens semi-directifs individuels avec cinq apprenants adultes australiens (fréquentant l'institution depuis plus de six mois), cinq enseignants de français, le directeur et le responsable des langues de l'enseignement catholique en Australie Occidentale $\left(\mathrm{CEO}^{2}\right)$. Nous exploiterons également les résultats d'une enquête de satisfaction réalisée par l'Alliance française.

8 Le but de notre démarche n'est pas de récolter des données en nombre mais de mettre en évidence la diversité (voire la divergence) des discours et de tenter de les interpréter. Les enquêtes concernent :

- le point de vue des enseignants sur leurs pratiques d'enseignement du FLE;

- le point de vue des apprenants destinataires de cet enseignement notamment sur

- les représentations de la langue française et sur les modalités de l'enseignement reçu ;

- le point de vue du directeur de l'institution sur les orientations didactiques choisies.

9 Notre expérience personnelle en tant qu'enseignante de FLE dans des Alliances françaises de pays différents et dans ce contexte particulier constituera également un apport réflexif non négligeable dans le processus d'interprétation.

\section{Le français en Australie : quelques données}

\section{Éléments sociolinguistiques et représentations du français}

10 À notre arrivée en Australie en 2007, nous avons été frappées par l'attirance de la population australienne pour la langue française. Notre expérience en tant qu'enseignante de français dans deux Alliances françaises du pays mais également nos contacts avec les Australiens nous permettent de dire qu'il existe sur ce continent un attrait certain pour la France et la langue française. On peut être surpris, dans un premier temps, de cette inclination envers le français et penser que la distance géographique séparant les deux pays (plus de 15000 kilomètres) n'est pas un facteur motivant pour apprendre la langue, dans le sens où les voyages dans le pays dont on apprend la langue peuvent se faire rares - tout en constituant une possible motivation par l'imaginaire. Comme le fait remarquer Dabène (1994:35), "en contexte extraeuropéen, le séjour dans le pays européen dont on enseigne la langue revêt une importance capitale [...]». Toutefois, qui ne rêve pas d'une contrée lointaine que l'on aime à 
imaginer exotique et différente? La distance, dans ce cas, peut se présenter comme un facteur d'attirance, pouvant combler une envie d'exotisme.

11 Le fait d'être attiré par telle ou telle langue repose, en général, sur les représentations sociales $^{3}$ qu'ont les individus sur les langues et sur les locuteurs de ces langues. Les discours des personnes interrogées dans le cadre de cette étude dévoilent une certaine admiration pour le français reposant sur une image très valorisée de la langue française. Par exemple, d'après le responsable des langues du CEO d'Australie occidentale, "French is viewed by many as a sophisticated European language that is desirable to learn ${ }^{4} »$. Par ailleurs, contrairement à l'italien ou au grec, le français n'est pas perçu comme une "langue d'immigrés» et selon la population australienne, les Français seraient venus s'installer en Australie pour des raisons autres que socio-économiques ou politiques (ce qui n'est pas le cas pour les Italiens et les Grecs qui ont trouvé refuge sur le continent australien lors de la dictature de Mussolini en Italie et suite au coup d'Etat de 1967 en Grèce). Par conséquent, ils n'apparaissent pas comme « des réfugiés politiques» et détiennent de ce fait un statut différent, plus valorisé. Enfin, numériquement, les Français sont très peu représentés, ce qui éloigne toute sensation d'être "envahi " par cette population. Ces perceptions des langues sont à mettre en relation avec l'histoire de l'Australie, qui a pratiqué une politique protectionniste (appelée White Australian Policy ${ }^{5}$ ) de 1901 à 1973. L'immigration a, de ce fait, longtemps été majoritairement britannique, ce qui a eu pour conséquence une certaine hostilité envers la diversité linguistique et envers les langues des "migrants " (autres que britanniques).

12 Il existe également des sentiments ambivalents envers les langues asiatiques, et ce, malgré la proximité géographique de l'Australie avec la Chine et le Japon et malgré les $10 \%$ de la population australienne venant d'Asie et d'importants enjeux politicoéconomiques (les placements des capitaux australiens en Asie représentent $60 \%$ de son investissement global). Ces réserves envers les langues asiatiques correspondent à des évènements historiques qui ont marqué le pays et qui semblent subsister dans les mentalités d'aujourd'hui, comme le "péril jaune $"^{6}$ et la tentative d'invasion de l'Australie par le Japon pendant la Seconde guerre mondiale. Pourtant, ces langues ont été introduites dans le système scolaire en 1994, ce qui a marqué une volonté politique explicite de la part de l'Australie de se rapprocher de ses voisins asiatiques. Toutefois, selon les résultats des entretiens, le japonais serait appris pour le commerce et le français pour la culture et le plaisir ; quant à l'indonésien et le chinois, ils sont perçus comme des langues qui «ne servent à rien $»^{7}$.

13 Ainsi, tous ces éléments profitent, d'une certaine façon, à la langue française et lui confèrent une position de force dans les mentalités et dans le système éducatif.

\section{Statut du français dans le monde éducatif ouest-australien}

L'attirance pour la langue française se traduit par une forte demande d'apprentissage de la part des Australiens et le français est choisi dans les différents types d'établissements éducatifs. Dans le système scolaire, le français fait partie du classement des langues étrangères prioritaires (avec le japonais, le chinois, l'indonésien, l'italien, et les langues indigènes) ce qui lui confère une place privilégiée si l'on considère le nombre de langues ${ }^{8}$ en présence sur le territoire australien. Le français connaît également une certaine popularité à l'université, étant la langue 
européenne ${ }^{9}$ majoritairement choisie. Ces étudiants de français se caractérisent par le fait qu'ils ne sont pas tous spécialistes, certains d'entre eux préparant des diplômes en droit ou en médecine. Enfin, l'intérêt pour la langue française en Australie occidentale se manifeste nettement à l'Alliance française Perth ${ }^{10}$ : d'une part les effectifs de fréquentation sont réguliers et importants (ce qui met en avant un net désir de la population locale d'apprendre ou de réapprendre le français) et, d'autre part, la clientèle qui côtoient ces cours est hétérogène (ce qui tend à indiquer que le français est une langue demandée par des personnes et pour des raisons très différentes).

Un élément historique important est à prendre en considération ici : l'enseignement du français en Australie relève d'une longue tradition britannique et le français a été la première langue étrangère enseignée dans le pays. Le début de l'enseignement du français appartient ainsi à l'histoire de la colonie à la fin du XIX ${ }^{e}$ siècle. Selon N. Bloomfield (chargée de recherches à University of Western Australia), la première école de la colonie établie à Port Jackson (Sydney aujourd'hui) proposait, entre autres, l'enseignement $d u$ français. Les colons, à forte majorité écossaise, ayant appris le français en Grande-Bretagne auraient continué d'enseigner le français dans leur nouvelle colonie. Cette tradition s'est perpétuée jusqu'à la Seconde guerre mondiale, puisque l'Australie était essentiellement peuplée de Britanniques. C'est avec la Guerre froide puis la mondialisation et l'arrivée d'autres types d'immigrés que l'enseignement des langues étrangères dans le système éducatif s'est largement diversifié.

$\mathrm{Au}$ regard de ce public et de cette demande hétérogènes, la question est de savoir si l'intérêt pour le français et le choix de son apprentissage se traduisent forcément par l'envie d'acquérir une compétence linguistique et communicative en langue française, ce qui correspond, en l'occurrence, à l'offre de l'Alliance française essentiellement centrée sur la compétence de communication.

\section{Hégémonie du communicatif : comment rater sa cible?}

\section{Choix des apprenants : un apprentissage pour le plaisir et une demande culturelle}

Le point soulevé ici rejoint la problématique du sens de l'apprentissage pour les apprenants. Les divers témoignages recueillis mettent à jour des idées et des représentations suggérant que ce n'est pas tellement la langue qui est en jeu mais une forme de proximité avec la France.

Ainsi, un sondage préparé par un stagiaire de l'Alliance française -comprenant 191 questionnaires $^{11}$ diffusés à la fin du premier trimestre- montre que les apprenants auraient choisi d'apprendre le français par intérêt personnel (52\%), pour les voyages (31\%) puis pour le caractère social et l'obligation professionnelle ( $7 \%$ pour les critères) ${ }^{12}$. Selon le directeur, l'intérêt pour la France est avant tout «l'attrait touristique de la France", plus dans une dimension culturelle que strictement linguistique. Une grande majorité des personnes interrogées apprennent le français sans nécessité fonctionnelle :

E: Why did you want to learn French? 
A1: (rires) Why, well I have been to France three times and I love here and and it's a hobby it's an interest and a passion for me now it's like doing a sport $^{13}$.

19 Cette situation se manifeste, premièrement, par le désir de proximité avec un locuteur natif français. L'enseignant « natif » constitue un facteur de motivation important dans une démarche d'apprentissage du français car il est perçu comme un gage de réussite (Castellotti, 2011 : 30). D'après notre expérience personnelle et lors des entretiens individuels, les apprenants de l'Alliance française de Perth affirment une inclination très favorable pour les enseignants natifs français et notamment pour leur accent, suggérant l'envie d'accéder à ce qui est « vrai » et « naturel ».

E : Et vous, vous pensez que c'est mieux d'avoir un enseignant natif?

A2 : Ici dans les écoles, il y a plein de profs anglais qui enseignent le français avec un accent et ils parlent anglais, c'est bien pour les enfants mais dans les écoles privées, on cherche des natifs.

$\mathrm{E}$ : Do you think it's important to have a native teacher?

A3: Oh yes it's good to learn the language with somebody who speaks it properly ${ }^{14}$.

20 Côtoyer un enseignant natif qui viendrait directement de France n'est ainsi pas seulement lié à ce qui est en français mais aussi à ce qui est français. Deuxièmement, la participation aux diverses activités proposées par l'Alliance française (débats, club de conversation, fête de la musique, etc.) constitue également un élément attractif pour les apprenants australiens, attraction qui reposerait sur deux points. D'une part, la distance géographique séparant la France de l'Australie rend l'Hexagone physiquement lointain mais encore plus désirable. En côtoyant l'Alliance, ces personnes s'en rapprochent, comme le dit cette apprenante :

A4: I love coming here, hearing people speaking French, looking at the French books in the library, and the posters too... I feel like being in France ${ }^{15}$.

21 D'autre part, il y a un aspect exotique à venir toutes les semaines apprendre le français et notamment, pendant les pauses, manger du fromage et boire du vin en discutant en français.

Dans le contexte anglophone australien, la question est donc de savoir quelles sont les raisons de cet attachement à la langue française, l'Australie ne présentant pas de lien identitaire local, ni de lien géographique avec l'Hexagone. Le français répondrait davantage à un désir d'accès à une certaine culture et s'expliquerait par un attachement culturel européen lointain: selon le directeur de l'Alliance, 50\% des effectifs sont d'origine britannique et certains apprenants déclarent que : "Europe is attractive to Australians because our roots, they were there as England was the mother country ${ }^{16}$ et "We are more Europeans than Asian, it's just obvious to learn French for $u s^{17} »$. Comme mentionné supra, le japonais se présente comme une «langue de raison "(ayant un rôle fonctionnel) tandis que le français est perçu comme une «langue de prestige», considéré comme une langue de culture, de distinction internationale et ayant parfois une dimension identitaire. Dans ce sens, la langue française est une langue emblématique, une sorte de langue internationale rêvée pour accéder à la culture française, et non pour des besoins communicatifs. De manière générale, l'attachement au français s'inscrit finalement peu dans une dimension linguistique et les personnes qui fréquentent l'Alliance française désirent avoir des 
choses en commun avec ce qui se passe en France, avec des Français, avec une langue qu'ils jugent mélodieuse, etc... Si on exclut une minorité de personnes qui apprennent le français à des fins strictement utilitaires et professionnelles, l'apprentissage du français est généralement motivé par le désir d'une forme de rapprochement avec la France par un attrait voire un amour pour la France ou/et la culture française au sens large comme le témoigne cet extrait d'entretien :

A5 : Je suis envoûtée par la France sa sa langue euh sa culture, son histoire et son peuple. Le français m'immerge dans un autre monde où mon esprit et mon âme comment dire ... sont bercés.

\section{Orientations formatives, offre et évaluations communicatives}

23 Le choix du français de la part des Australiens répond à une demande plus culturelle que linguistique et à des finalités de plaisir. Toutefois, l'analyse des orientations didactiques choisies dans l'institution concernée révèle que, du point de vue de l'offre en matière d'enseignement du FLE, les choses sont envisagées différemment. En effet, les cours de français proposés s'alignent sur une perspective largement communicative, souvent réduite à des objectifs fonctionnels, qui se manifeste à plusieurs niveaux.

\section{Le choix du manuel}

L'apprentissage de la langue est organisé par unités capitalisables à travers un manuel de FLE généraliste Alter Ego inspiré du Cadre européen commun de référence pour les langues (désormais CECR ou Cadre) et initiant à un " français de survie ", dans une perspective très explicitement communicative ${ }^{18}$. Selon le directeur, le critère ayant principalement présidé au choix du manuel serait celui de sa récente date de publication et non le fait qu'il correspond à la demande, à ce pour quoi les apprenants s'inscrivent dans les cours de français.

D1 : On a choisi Alter Ego c'est-à-dire moi et l'équipe pédagogique, on a pris les quatre ou cinq dernières méthodes FLE parues, c'est important de travailler avec du matériel récent et on n'en a éliminé etc. et puis on est arrivés à une.

Le choix d'un manuel de FLE généraliste renverrait à une certaine tradition selon laquelle le foyer de la conception du matériel de FLE reste localisé en France. En créant des méthodes répondant à des besoins généraux pressentis définis a priori, les maisons d'édition conçoivent et vendent du matériel pédagogique susceptible de satisfaire, de manière globale, un public considéré comme homogène, ayant les mêmes besoins langagiers et les mêmes objectifs d'apprentissage quels que soient le projet, le lieu, le temps, la situation dans laquelle ils apprennent le français. La provenance et les caractéristiques du manuel utilisé pour le FLE dans cette Alliance ne semblent pas être questionnés par le directeur qui reçoit régulièrement des ouvrages FLE des maisons d'édition françaises, conditionnant ainsi son choix. Les enseignants interrogés, eux, s'accordent à dire que le matériel répond aux besoins des apprenants :

E : Est-ce tu penses que Alter Ego correspond aux besoins des étudiants? Ens1: Oui, étant donné qu'on fait beaucoup de français communicatif oui ce sont des choses qu'ils vont pouvoir utiliser, dans l'ensemble ils seront bien préparés. 
Ens2: Oui je pense je pense que ça correspond à ce qu'il faut enseigner mais j'utilise la vidéo de temps en temps je l'utilise pour l'oral. basé essentiellement sur la progression d'Alter Ego mais qui pourrait être plus adapté à ce type de public. Le communicatif est perçu comme une évidence et cette situation crée un certain hiatus entre l'offre et la demande, parfois verbalisé par les apprenants :

A5: Something I would like more, it's about vocabulary and... so to travel in France it's not that relevant in the book for me or learn more about France [...] Things that you will use as a traveller, as a tourist and the thing about masculine and feminine we are not going to use that, it should be at the end of the book. ${ }^{19}$

A6: Learning the different types of French food or accommodation or something more useful. ${ }^{20}$

Ces témoignages montrent bien que le communicatif n'est pas en adéquation avec à ce que ces individus recherchent dans leur choix d'apprendre le français.

\section{Les évaluations sommatives et d'orientation}

Au début de la formation, les futurs apprenants sont soumis à un test linguistique afin de mesurer leurs acquis en français et de déterminer leur niveau de compétence. Aucune information concernant, par exemple, le projet des apprenants ou leur biographie langagière n'est requise, toutefois le but de cette évaluation est d'orienter les apprenants dans leur apprentissage. Une évaluation sommative est également proposée par l'Alliance avec la validation des compétences présentes dans les certifications DELF ${ }^{21} \mathrm{CEFP}^{22}$, $\mathrm{TCF}^{23}$ et $\mathrm{TEF}^{24}$, basées sur le CECR. Toutefois, ses évaluations à caractère linguistique ou communicatif ne correspondent pas non plus aux apprenants qui ne sont pas demandeurs de certification, comme le directeur le dit luimême :

D2: (...) C'est intéressant parce que ça rejoint le problème des examens, des tests etc... justement ces Australiens qui viennent apprendre le français ne veulent pas entendre parler d'examen, d'évaluations ni de tests ni de diplôme, ça les stresse, ils ne viennent pas là pour ça.

Cette situation soulève une fois encore le paradoxe entre la suprématie du communicatif de la formation en décalage avec une demande plus culturelle.

\section{L'omniprésente référence au CECR}

La référence au CECR, déjà mentionné dans le manuel utilisé et les certifications proposées par l'institution, est clairement mise en avant à plusieurs reprises sur le site Internet de l'Alliance française venant ainsi renforcer l'hégémonie de l'approche communicative :

Courses are modeled after the Common European Framework of References for Languages and comply with the criteria of the DELF and DALF diplomas. ${ }^{25}$ 
Class levels are based on the 6 stages outlined by the Common European Framework of Reference (CEFR) for Languages. At the end of each level, students can sit an official exam recognised by the French Government. ${ }^{26}$ http://www.afperth.com.au/language-centre/adult-courses

30 Considéré comme LA norme didactique, le modèle de référence par excellence pour enseigner / apprendre le français ${ }^{27}$, le Cadre et son instrumentalisation constituerait, selon l'institution, un bon argument de vente. Toutefois, son usage pose question dans le contexte australien dans la mesure où il constitue initialement et avant tout un instrument politique, culturel et économique conçu afin de répondre «aux besoins d'une Europe multiculturelle et multilinguistique en développant sensiblement la capacité des Européens à communiquer entre eux par-delà les frontières linguistiques et culturels » (Conseil de l'Europe, $2001: 10$ ).

31 Or, le CECR, comparé par Coste (2014) à un couteau suisse - instrument robuste mais à usages multiples -, n'est qu'un modèle parmi d'autres qui se veut modulable et malléable impliquant une mise en relation avec un contexte national, régional, local pour prendre place et sens (2007). Castellotti \& Nishiyama (2011) prolongent cette réflexion en interrogeant la pertinence et la cohérence de la mise en œuvre de possibles formes de contextualisation du CECR en Asie du Sud-Est (exemples et réflexions qui seraient transposables au-delà de cette région). En dehors de ces objectifs politiques, l'usage du cadre réduit à ses dimensions strictement techniques et méthodologiques est-il vraiment approprié ?

On pourrait également interroger le rôle de la recherche en didactique du FLE dans le sens où elle reste largement centrée sur une orientation communicative et pragmatiste, qu'elle pense comme une évidence et ne questionne donc pas, ce qui accentue encore les effets d'hégémonie- tendance dénoncée par Huver : $(2014: 145):$ «la prise en compte de la variation et des 'normes endogènes' semble valoir au niveau linguistique et culturel, mais pas au niveau proprement didactique ».

\section{Quelques raisons à ces divergences constatées}

Dans le cas présenté ici, l'offre de formation ne questionne pas les raisons pour lesquelles ces personnes veulent apprendre le français. Les apprenants sont placés dans des classes en fonction d'une estimation de leur niveau. Au cours de l'apprentissage, bien que les besoins soient selon Porcher des « réalités dites fondantes" (1995: 25) et qu'ils se modifient au cours de la formation, aucune analyse des besoins n'est envisagée avant et durant l'apprentissage. L'institution éducative n'élabore pas d'objectifs d'apprentissage en lien avec les besoins des apprenants et a ses propres objectifs illustrés par des contenus d'apprentissage définis. En d'autres termes, les apprenants suivront le programme préalablement conçu qui se présente comme un apprentissage du français à visée communicative. Cette situation soulève ainsi le problème de la souplesse et de la flexibilité de ce type de structure dont la pédagogie et l'approche systémique ne sont pas centrées sur l'apprenant mais sur l'institution elle-même. Ce système, de plus en plus généralisé, est régi par des tendances pédagogiques basées sur la construction d'objectifs élaborés d'avance et sans prise en compte des besoins des apprenants ni de l'avis des enseignants (Idem), qui ne semblent pas questionner non plus la visée communicative. Cela suggère également que l'offre est définie au préalable 
et que toute négociation entre les apprenants et enseignants est limitée voire inexistante. tellement importante qu'elle occulte la prise en compte de la diversité des publics et ne permet pas la mise en place d'une contextualisation de l'enseignement du français (dans ce contexte ou dans d'autres contextes). Comme nous l'avons évoqué supra, cette situation peut s'expliquer par la conjonction de différentes raisons comme :

- le poids des maisons d'édition françaises et de la production de manuels universalistes à vocation uniquement communicative ;

- l'importance des certifications existantes et de l'injonction même à la certification ;

- l'utilisation du CECR par les instances françaises comme un outil de leur politique d'influence ;

- des recherches qui ne remettent pas en question cette orientation communicative et qui contribuent même à sa diffusion, voire à son hégémonie (par le biais des recherches, des thèses encadrées et des missions de formation assurées).

D'une certaine façon, on pourrait se demander dans quelle mesure cette situation a un lien avec ce qui constitue les grands traits de l'enseignement du français dans le monde, c'est-à-dire le champ du FLE en lui-même. Le FLE est né dans un contexte d'après-guerre marqué par une volonté politique de diffuser la langue française dans le monde et dans une moindre mesure en France (pour le public migrant). Ce désir d'étendre le français répondait, au lendemain de la Seconde guerre mondiale, à la nécessité d'intégrer de nouveau la place de la France sur la scène internationale. Le FLE a pris forme de façon prépondérante dans une perspective de diffusion du français rendue possible par des moyens mis en œuvre afin de favoriser son expansion. Cette caractéristique fondatrice du champ parait substantielle pour comprendre et expliquer la situation actuelle de l'enseignement du français dans le monde. Il apparaît que le français hors de France est toujours pensé en termes de diffusion malgré l'évolution de la situation sociolinguistique internationale et que le rôle de la France repose essentiellement sur l'action de répandre sa langue (et ses valeurs) de façon universaliste sans prendre en compte les environnements concernés. Il existerait donc un malentendu quant à la question de diffusion du français, essentiellement perçue dans son aspect linguistique et se traduisant par l'enseignement de la langue. Or, il s'agit de la diffusion du français dans son ensemble, c'est-à-dire de ses représentations et de ses valeurs à la fois linguistiques et culturelles qu'il faudrait peut-être considérer dans le contexte anglophone australien.

Cette étude de $\operatorname{cas}^{28}$ pointe du doigt un manque d'articulation entre l'offre et la demande et met à jour non seulement l'absence de toute contextualisation de l'enseignement du français mais aussi la volonté d'imposer, malgré d'éventuelles demandes locales, un enseignement français visant une compétence de communication. Toutefois, comme mentionné supra, ce n'est pas seulement l'institution de formation la grande accusée mais c'est tout un système qui fonctionne sur la base de cette évidence non interrogée qu'est le communicatif et qui ne prend pas en compte le sens que peut avoir l'apprentissage du français pour les apprenants.

On pourrait, en outre, se demander si les apprenants eux-mêmes savent exprimer leurs projets et leurs besoins et par conséquent le type de cours qu'ils désirent recevoir. Face à ce phénomène généralisé, la recherche en didactique des langues devrait, comme le suggère Castellotti (2014:122), "[penser], fondamentalement, la diversité humaine 
constitutive des situations d'appropriation (...) en travaillant leur historicité et en incluant le/la chercheur-e dans cette contextualisation et cette historicisation ».

\section{BIBLIOGRAPHIE}

Berthet. A., Hugot, C. et al (2006). Alter Ego. Paris : Hachette FLE.

Besse, H. (2009). Pourquoi apprend-on encore le français en tant que langue étrangère ? Revue japonaise de didactique du français, $\mathrm{n}^{\circ} 1$.

Blanchet, P. \& Chardenet, P. (dir.) (2011). Guide pour la recherche en didactique des langues et des cultures : approches contextualisées. Paris : Eds des Archives contemporaines.

Blanchet, P., Moore, D. \& Asselah Rahal, S. (dir.) (2009). Perspectives pour une didactique des langues contextualisée. Paris : Eds des archives contemporaines.

Castellotti, V. (2014). Contexte, contextualisation, cultures éducatives. Quels usages ? Pour quelles orientations de la recherche en DDL ? Actes du colloque international Contexte global et contextes locaux : tensions, convergences et enjeux didactiques des langues.

Castellotti, V. (2011). Natif, non natif ou plurilingue : dénativiser l'enseignement des langues? Actes du colloque Hétérogénéité et variation : quels objets socio-linguistiques et didactiques aujourd'hui?

Castellotti, V. \& Moore, D. (2009). Contextualisation et universalisme, quelle didactique des langues pour le XXI ${ }^{\mathrm{e}}$ siècle ? Perspectives pour une didactique des langues contextualisée.

Castellotti. V. \& Nishiyama. J. N. (2011). Contextualiser le CECRL ?, Contextualisations du CECR. Le cas de l'Asie du Sud-Est. Le français dans le monde, Recherches et applications, $\mathrm{n}^{\circ} 50$.

Conseil de l'Europe (2001). Cadre européen commun de référence pour les langues : Apprendre, enseigner, évaluer. Paris : Didier.

Coste, D. (1984). Aspects d'une politique de diffusion du français langue étrangère depuis 1945. Paris : Hatier.

Coste, D. (2014). De la conception aux usages : CECR et couteau suisse. Les Cahiers du GEPE. Disponible en ligne. http://www.cahiersdugepe.fr/index2628.php

Coste, D. (2007) : Le Cadre européen commun de référence pour les langues. Contextualisation et/ou standardisation? Disponible en ligne. http://www.francparler.org/dossiers/ cecr_perspectives.htm

Coste, D. (2006). Pluralité des langues, diversité des contextes : quels enjeux pour le français? In Castellotti \& Chalabi (dir.). Le français langue étrangère et seconde, des paysages didactiques en contexte. Paris : L'Harmattan.

Dabene, L. (1994). Repères sociolinguistiques pour l'enseignement des langues. Paris : Hachette.

Huver, E. (2014b). « Parler de la diversité sans “contexte" ?», Colloque international Contexte global et contextes locaux : tensions, convergences et enjeux en didactique des langues, Paris, 23-25 janvier 2014. 140-154. 
http://fipf.org/sites/fipf.org/files/

actescolloquecontexteglobaletcontexteslocauxsorbonnenouvelleparis32014.pdf.

Moscovici, S. (1961). La psychanalyse, son image et son public. Paris : Presses Universitaires de France.

Nettelbeck, C. (1990). The Alliance Française in Australia 1890-1990. Clayton : Institute for the Study of French-Australian Relations.

Porcher, L. (1995). Le français langue étrangère. Paris : Hachette Education.

Porquier, R \& PY, B. (2008). Apprentissage d'une langue étrangère : contextes et discours. Paris : Didier.

Richterich, R. \& Chancerel, J.L. (1977). L'identification des besoins des adultes apprenant une langue étrangère. Conseil de l'Europe. Paris : Hatier.

Richterich R. (1986). Besoins langagiers et objectifs d'apprentissage. Paris : Hachette. Collection Recherches-Applications.

Truchot, C. (2014). Le contexte politique et économique du CECR et les politiques européennes. Les Cahiers du GEPE. Disponible en ligne. http://www.cahiersdugepe.fr/index2685.php

Zarate, G. (1997). Les représentations en didactique des langues et culture. Notions en Questions, $\mathrm{n}^{\mathrm{o}}$ 2. Paris : Didier Erudition.

\section{NOTES}

1. Le cas analysé ici permettra d'illustrer des tendances plus générales et ne représente qu'un exemple parmi d'autres.

2. Catholic Education Office

3. Nous comprenons "représentations sociales" comme une "famille de concepts - idéologie, vision du monde, idée-force, mythe, utopie - qui se réfèrent tous à une élaboration théorique censée refléter les rapports sociaux tout en contribuant à les édifier " (Moscovici, 1961 : 300).

4. Le français est vu par beaucoup comme une langue européenne sophistiquée qu'il est souhaitable d'apprendre.

5. Politique de l'Australie blanche

6. L'immigration asiatique a longtemps été perçue comme une menace pour l'économie et l'unité culturelle et a provoqué la promulgation de lois strictes sur l'immigration jusqu'à la fin de la politique de «l'Australie blanche » (dans les années 70).

7. La question était What do you think about languages like Indonesian, Chinese or Japanese ? (Que pensez-vous des langues comme l'indonésien, le chinois ou le japonais ?)

8. Il y aurait environ 240 langues autres que l'anglais parlées en 2012 en Australie selon l' Australian Bureau of Statistics.

9. Les autres langues européennes étant l'allemand et l'italien.

10. Le réseau des Alliance françaises en Australie s'est constitué tôt: la première à ouvrir ces portes fut celle de Melbourne en 1889, soit six ans après celle de Paris (Nettelbeck, 1990). Aujourd'hui, selon la Fédération des Alliances françaises en Australie, il y aurait 31 associations dont cinq proposant des cours de français.

11. Sondage incluant différents types de questions (questions ouvertes, fermées et mixtes).

12. La question posée étant Why did you enrol in French lessons? (Pourquoi vous êtes-vous inscrit à des classes de français?).

13. E: Pourquoi vouliez-vous apprendre le français?

A1 : (rires) pourquoi, bon je suis allée en France trois fois et j'adore et et c'est un passe-temps c'est un 
intérêt personnel et une passion pour moi maintenant c'est comme faire un sport. (Entretien individuel apprenant)

14. E : Pensez-vous que c'est important d'avoir un enseignant natif ?

A3: Oh oui c'est bien d'apprendre une langue avec quelqu'un qui la parle correctement.

15. J'adore venir ici, entendre des gens parler français, regarder les livres français dans la bibliothèque et les affiches aussi... j'ai l'impression d'être en France.

16. L'Europe est pour les Australiens parce que nos racines, elles viennent de là-bas comme l'Angleterre était la mère-patrie.

17. Nous sommes plus Européens qu'Asiatiques, c'est évident pour nous d'apprendre le français.

18. "L'approche retenue permet [à l'apprenant] d'acquérir des compétences de communication écrite et orale, de compréhension et d'expression, à travers des tâches communicatives" (Berthet, Hugot et al, 2006 : 3). On parle aussi de "développer des savoir-faire et savoir-être indispensables à toute communication réussie" et on précise que "Chaque leçon est structurée par les objectifs communicatifs" (Idem : 4).

19. J'aimerais apprendre des choses ou du vocabulaire... plus comme pour voyager ce n'est pas vraiment dans le livre pour moi ou apprendre des choses sur la France. Les choses sur le masculin et le féminin on ne va pas l'utiliser.

20. Apprendre sur les différentes spécialités culinaires françaises ou sur le logement ou des choses plus utiles.

21. Diplôme d'études en langue française

22. Certificat d'études de français pratique

23. Test de connaissance du français

24. Test d'évaluation de français

25. Les cours sont calqués sur le Cadre européen commun de référence pour les langues et respectent les critères des diplômes DELF et DALF.

26. Les niveaux de classe sont basés sur les six étapes décrites par le Cadre européen commun de référence (CECR) pour les langues. A la fin de chaque niveau, les élèves peuvent passer un examen officiel reconnu par le gouvernement français.

27. Malgré les rappels réguliers des auteurs sur le fait que le CECR n'est pas une méthode ou une norme.

28. Ce terrain ne constitue qu'un exemple et, dans une dimension plus large, les interrogations au sujet de la contextualisation de l'enseignement du français se posent de façon plus générale (par exemple, les articles de V. Castellotti (2014): Contexte, contextualisation, cultures éducatives. Quels usages? Pour quelles orientations de la recherche en DDL ? et de E. Huver (2014): Quand contexte homogénéise la diversité OU : Parler de la diversité sans contexte ?)

\section{RÉSUMÉS}

Cet article se base sur une recherche interprétative s'appuyant sur deux points de vue : celui la demande et celui de l'offre en matière d'apprentissage/enseignement du FLE dans un microcontexte. Par un jeu de regards croisés, nous mettrons en évidence un manque d'harmonisation entre le discours des apprenants et les approches didactiques mises en œuvre pour enseigner le français puis nous interrogerons quelques aspects de cet enseignement avant de proposer d'éventuelles formes de contextualisation. 
This article is based on interpretative research using two viewpoints: the supply and the demand of learning/teaching French as a Foreign Language in a micro-context. Using different perspectives, we will be able to highlight a lack of harmonization between the learners' discourse and the teaching approaches in French language classes. We will examine some of these aspects before proposing any form of contextualization.

\section{INDEX}

Keywords : western Australia, learners, language teaching, french as a foreign language, needs analysis, contextualization

Mots-clés : Australie occidentale, apprenants, orientations didactiques, français langue étrangère, analyse des besoins, contextualisation

\section{AUTEURS}

\section{CÉLINE DOUCET}

Céline Doucet est maître de conférences à l'Université Edith Cowan en Australie occidentale. Membre de l'équipe CREATEC (Université Edith Cowan) et associée à l'E.A. 4246 PREFics (Université de Rennes 2 et de F. Rabelais de Tours). Ses recherches s'inscrivent dans le domaine de la didactique du français langue étrangère et s'intéressent plus particulièrement à la contextualisation de l'enseignement du français hors de France.

Courriel : celine.doucet[at]ecu.edu.au

Toile : http://dynadiv.univ-tours.fr/membres/membres-175345.kjsp

Adresse : Edith Cowan University, School of Communications and Arts, 2 Bradford Street, Mount Lawley, 6050, WA, Australie.

\section{EDITH COWAN}

University, Mount Lawley, Australie 